\title{
CORRELAÇÃO ENTRE O COEFICIENTE DE DIFUSÃO E A CORRENTE ELÉTRICA DURANTE ENSAIO MULTIREGIME DE MIGRAÇÃO DE CLORETOS
}

\author{
Correlation between diffusion coefficient and electric current \\ during chloride migration multiregime test
}

\author{
Ronaldo Alves de Medeiros-Junior ${ }^{1}$, Maryangela Geimba de Lima ${ }^{2}$
}

Recebido em 13 de outubro de 2014; recebido para revisão em 18 de outubro de 2014; aceito em 11 de dezembro de 2014; disponível on-line em 12 de dezembro de 2014.

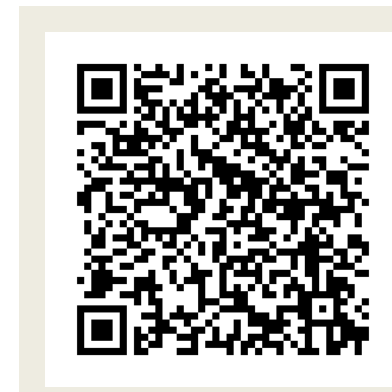

PALAVRAS CHAVE:

Estruturas de concreto armado;

Cloreto;

Coeficiente de difusão;

Corrente elétrica;

Cimentos.

\section{KEYWORDS:}

Reinforced concrete structures;

Chloride;

Diffusion coefficient;

Electric current;

Cements.
RESUMO: Os cloretos são extremamente agressivos as estruturas de concreto armado, pois induzem o início da reação de corrosão das armaduras. Boa parte dos modelos disponíveis para predizer a penetração dos cloretos no concreto utiliza como parâmetro de entrada o coeficiente de difusão de cloretos. Este artigo propõe uma alternativa para a determinação do coeficiente de difusão durante o ensaio de migração de cloretos: o uso da corrente elétrica. Para tal, diferentes amostras de concreto foram moldadas, com quatro tipos de cimentos brasileiros. Foram realizados ensaios de resistência à compressão e resistividade elétrica superficial para caracterizar essas amostras de concreto. Posteriormente, o ensaio multiregime de migração de cloretos foi executado para determinação dos coeficientes de difusão efetivo e aparente, paralelamente ao monitoramento da corrente elétrica durante o ensaio. Os resultados mostraram boas correlações entre os dados. Para as condições utilizadas neste artigo, o coeficiente de difusão efetivo é cerca de 4,2 vezes maior que o coeficiente aparente. Além disso, os picos de corrente variam de acordo com o tipo de cimento utilizado, sendo menor para as amostras com cimentos ricos em adições. As equações de ajuste do tipo linear encontradas permitem o uso de uma ferramenta alternativa para determinação dos coeficientes de difusão durante o ensaio de migração de cloretos.

ABSTRACT: Chlorides are extremely aggressive to reinforced concrete structures, as they induce the onset of the reinforcement corrosion reaction. Much of the available models for predicting the chloride penetration in concrete uses the chloride diffusion coefficient as an input parameter. This article proposes an alternative to the determination of the diffusion coefficient during the chloride migration test: the use of electric current. Therefore, different concrete samples were cast with four types of Brazilian cement. Testing of compressive strength and electrical resistivity were performed to characterize these concrete samples. Subsequently, the chloride migration multiregime test was performed to determine the effective and apparent diffusion coefficients. In parallel, the electric current was monitored during the test. The results showed good correlation between the data. For the conditions used in this paper, the effective diffusion coefficient is about 4.2 times higher than the apparent coefficient. Also, current peaks vary with the type of cement used. These peaks are smaller for cements with high content of additions. Adjustment equations (linear) found here allow the use of an alternative tool for determination of the diffusion coefficients during the chloride migration test.

\footnotetext{
* Contato com os autores:

${ }^{1}$ e-mail : ronaldodemedeirosjr@yahoo.com.br ( R. A. de Medeiros-Junior )

Doutor em Eng. Civil, Pós-doutorando, Instituto Tecnológico de Aeronáutica (ITA).

2 e-mail : magdlima@gmail.com (M. G. de Lima)

Doutor em Eng. Civil, Profa., Instituto Tecnológico de Aeronáutica (ITA).
} 


\section{INTRODUÇÃO}

A corrosão das armaduras devido à penetração de cloretos é o processo de degradação com maior ocorrência nas estruturas de concreto armado expostas em ambiente marinho. Mesmo as obras construídas distantes da linha da costa, mas situadas na chamada zona de atmosfera marinha, continuam a receber a ação dos cloretos, o que é mais importante ao longo das primeiras centenas de metros além da costa (MEIRA, 2004). Portanto, estudos voltados à durabilidade do concreto armado frente ao ataque de cloretos são ainda mais importantes em países com urbanização intensa na região costeira, como é o caso do Brasil.

Soma-se a esse efeito a necessidade crescente de uso de estruturas de concreto offshore em países em desenvolvimento, tais como portos, plataformas de petróleo e aeroportos, onde o concreto se caracteriza como material de uso fundamental (MEDEIROS-JUNIOR, 2014).

A corrosão das armaduras é uma reação que gera produtos expansivos. Esses produtos ocupam um volume significativamente superior ao volume original das armaduras, elevando as tensões de tração no interior do concreto. Em paralelo, a corrosão ocasiona a redução da seção transversal do aço, diminuindo também a aderência entre a armadura e o concreto. Tal comportamento provoca o aparecimento de fissuras e rachaduras, e o posterior destacamento da camada de cobrimento, comprometendo o dimensionamento estrutural (ANDRADE, 1992; HELENE, 1993; MEHTA e MONTEIRO, 2005).

O tempo de início da corrosão é influenciado principalmente pela qualidade do concreto, por meio do controle de parâmetros relacionados à permeabilidade do concreto quanto à penetração dos cloretos, e à espessura da camada de cobrimento do concreto, que protege as armaduras.

Dentro deste contexto, segundo Andrade e D'Andréa (2010), projetar estruturas de concreto a partir do conceito de durabilidade vem sendo assunto de demanda crescente na construção civil.
Tal afirmação é sustentada devido às recomendações das normas existentes se mostrarem diversas vezes insuficientes em garantir a vida útil projetada para as estruturas de concreto armado em meios ambientes agressivos (MEDEIROS-JUNIOR, 2011), e, também, devido à necessidade de otimizar a utilização de recursos naturais, em ordem de aprimorar a sustentabilidade da utilização do concreto.

Considerando a quantificação do ingresso de cloretos no concreto, de forma prática, os perfis obtidos em estruturas existentes se ajustam de modo satisfatório ao fenômeno de difusão (RAMLI e TABASSI, 2012; REDDY, EDOUARD e SOBHAN, 2013).

Porém, observa-se que frequentemente é negligenciado o fato de existirem pelo menos dois tipos de coeficiente de difusão: um obtido por meio de experimentos em um estado estacionário (ANDRADE, 1993), chamado de coeficiente de difusão efetivo $\left(D_{e f}\right)$; e outro a partir de um estado não estacionário (ANDRADE et al., 1994), também conhecido como coeficiente de difusão aparente $\left(D_{a p}\right)$ (CASTELLOTE, ANDRADE e ALONSO, 2001).

Segundo Andrade e Whiting (1996), o $D_{a p}$ considera que reações de ligação entre os cloretos e a matriz cimentícia do concreto ocorrem simultaneamente à difusão desses íons. Uma forma comum de obter $D_{a p}$ é através da medição do perfil de cloretos em concreto submetido à solução de cloretos - conhecido como ponding test - por um período mínimo de 90 dias (ATKINSON e NICKERSON, 1984).

Por outro lado, $D_{e f}$ é obtido considerando que o concreto já está saturado com cloretos, ou seja, não são consideradas reações de ligação dos cloretos durante a difusão. Esse coeficiente é determinado principalmente através de células de difusão ou migração (ANDRADE, 1993).

A Norma Espanhola UNE 83987 (2012) sugere um método bastante atrativo para determinação de ambos, $D_{e f}$ e $D_{a p}$, através do ensaio multiregime de migração de cloretos. Tendo isso em vista, o objetivo deste artigo é buscar correlações entre esses coeficientes de difusão obtidos pelo método de ensaio de migração, com a corrente elétrica que atravessa o 
concreto durante o mesmo ensaio, uma vez que a corrente é facilmente medida por intermédio de um multímetro, diferentemente do acompanhamento da evolução da concentração de cloretos nas soluções do ensaio. Sendo assim, esse artigo procurou investigar uma ferramenta alternativa para medir os coeficientes de difusão de cloretos no concreto, com a finalidade de facilitar o método de ensaio e reduzir o tempo de determinação dessas variáveis importantes para a modelagem de vida útil.

\section{PROGRAMA EXPERIMENTAL}

Neste artigo, o programa experimental realizado nas amostras de concreto pode ser resumido da seguinte maneira: moldagem das amostras, ensaios de caracterização do concreto, ensaio multiregime de migração de cloretos.

A Tabela 1 mostra a proporção dos materiais utilizados nas amostras de concreto. Foram usados 4 tipos de cimento: 1- CPII-F cimento Portland composto com adição de fíler calcário (NBR 11578, ABNT 1991); 2- CPIIIRS cimento Portland de alto-forno (NBR 5735, ABNT 1991) e resistente a sulfatos (NBR 5737, ABNT 1992); 3- CPIV cimento Portland pozolânico (NBR 5736, ABNT 1991); e 4- CPV cimento Portland de alta resistência inicial (NBR 5733, ABNT 1991).

Como agregado miúdo, areia natural (proveniente de rio) disponível comercialmente na cidade de São José dos Campos - SP foi utilizada, com um tamanho máximo do agregado de 2,36 $\mathrm{mm}$, absorção de água de 0,97\%, massa específica de $2,91 \mathrm{~kg} / \mathrm{dm}^{3}$, e material fino que passa na peneira $75 \mu \mathrm{m}$ de 1,50\%. Esses ensaios foram realizados de acordo com as normas da Associação Brasileira de Normas Técnica (ABNT): NBR NM 248 (2003); NBR NM 30 (2001); NBR NM 52 (2009); e NBR NM 46 (2003).

O agregado graúdo utilizado foi brita comercial com dimensão máxima de 9,5 mm, absorção de água de 1,70\%, massa específica aparente de $2,64 \mathrm{~kg} / \mathrm{dm}^{3}$, e material fino que passa na peneira $75 \mu \mathrm{m}$ de $2,20 \%$, de acordo com as normas: NBR NM 248 (2003); NBR NM 53 (2009); e NBR NM 46 (2003).

De acordo com a Tabela 1, o mesmo traço 1:1,4:2,1 (cimento:areia:brita), em materiais secos, foi utilizado para todas as amostras. Não foi utilizado nenhum tipo de aditivo químico.

Corpos cilíndricos de $\varnothing 100$ mm x 200 $\mathrm{mm}$ de comprimento foram preparados para os ensaios de laboratório, com exceção do ensaio de resistividade elétrica superficial, no qual foram utilizados corpos de prova cúbicos, conforme será abordado no item 2.2 deste artigo. Após a moldagem, as amostras permaneceram em câmara úmida (temperatura $22 \pm 3^{\circ} \mathrm{C}$ e umidade relativa $\pm 65 \%$ ) para cura saturada durante um período de 28 dias para os ensaios de resistência à compressão e resistividade elétrica superficial, e 91 dias para o ensaio multiregime de migração de cloretos. A seguir, as principais informações a respeito destes ensaios serão apresentadas.

\begin{tabular}{cccccccc}
\hline \multicolumn{7}{c}{ TABELA 1: Proporção dos materiais das amostras de concreto. } \\
Código & $\begin{array}{c}\text { Tipo de } \\
\text { cimento }\end{array}$ & $\begin{array}{c}\text { Classe } \\
\text { cimento }\end{array}$ & $\begin{array}{c}\text { Teor de } \\
\text { argamassa } \\
\mathbf{( \% )}\end{array}$ & $\begin{array}{c}\text { Cimento } \\
\mathbf{( k g )}\end{array}$ & $\begin{array}{c}\text { Areia } \\
\mathbf{( k g )}\end{array}$ & $\begin{array}{c}\text { Brita } \\
\text { (Kg) }\end{array}$ & $\begin{array}{c}\text { Relação } \\
\text { a/c }\end{array}$ \\
\hline IIF04-32 & CPII-F & 32 & 53 & 1,00 & 1,40 & 2,10 & 0,4 \\
\hline IIF05-32 & CPII-F & 32 & 53 & 1,00 & 1,40 & 2,10 & 0,5 \\
\hline IIIRS04-40 & CPIIIRS & 40 & 53 & 1,00 & 1,40 & 2,10 & 0,4 \\
\hline IIIRS05-40 & CPIIIRS & 40 & 53 & 1,00 & 1,40 & 2,10 & 0,5 \\
\hline IIIRS06-40 & CPIIIRS & 40 & 53 & 1,00 & 1,40 & 2,10 & 0,6 \\
\hline IV04-32 & CPIV & 32 & 53 & 1,00 & 1,40 & 2,10 & 0,4 \\
\hline IV05-32 & CPIV & 32 & 53 & 1,00 & 1,40 & 2,10 & 0,5 \\
\hline IV06-32 & CPIV & 32 & 53 & 1,00 & 1,40 & 2,10 & 0,6 \\
\hline V04-ARI & CPV & ARI & 53 & 1,00 & 1,40 & 2,10 & 0,4 \\
\hline V05-ARI & CPV & ARI & 53 & 1,00 & 1,40 & 2,10 & 0,5 \\
\hline V06-ARI & CPV & ARI & 53 & 1,00 & 1,40 & 2,10 & 0,6 \\
\hline & & & & & FONTE: AUTORES (2014).
\end{tabular}




\subsection{RESISTÊNCIA À COMPRESSÃO}

A resistência à compressão de cada corpo de prova foi determinada segundo a Norma Brasileira NBR 5739 (2007). Para tal, foi utilizada uma prensa hidráulica, equipada com sistema de pratos com 200 toneladas de capacidade para aplicação de carga. Os ensaios foram realizados aos 28 dias de idade, e foram utilizados 2 corpos de prova para cada uma das dosagens investigadas, totalizando 22 corpos de prova.

\subsection{RESISTIVIDADE ELÉTRICA SUPERFICIAL (RES)}

O método dos quatro pontos, também conhecido como método de Wenner, foi executado para obtenção da RES das amostras aos 28 dias de idade. Corpos de prova cúbicos (arestas de $250 \mathrm{~mm}$ ) em condição de saturação em água foram ensaiados. A definição das dimensões dos corpos de prova foi baseada na experiência de ensaios discutida por Gowers e Millard (1999). Segundo recomendações desses autores, para realização de medidas de RES no concreto, alguns parâmetros geométricos devem ser respeitados para a obtenção de leituras precisas nos equipamentos empregados. Essas recomendações garantem que as linhas de correntes geradas pelo equipamento no interior do concreto se fechem e, dessa forma, não ocasionem alterações nos valores de RES obtidos. Segundo o estudo citado, para as condições de ensaio (tipo de equipamento) utilizadas neste artigo, as dimensões mínimas dos corpos de prova devem ser de $200 \mathrm{~mm}$. Portanto, a favor da segurança, foram utilizadas dimensões iguais a $250 \mathrm{~mm}$ para os corpos de prova cúbicos deste artigo. Uma dimensão maior não foi usada devido a dificuldades operacionais resultantes de um corpo de prova maior que o mencionado, como peso para locomoção e transporte das amostras.

Um equipamento disponível comercialmente foi utilizado para o ensaio. 0 equipamento possui quatro contatos espaçados a igual distância $(\mathrm{d}=50 \mathrm{~mm}$ ). Ao ser posicionado sobre a surpefície do corpo de prova, uma corrente elétrica é aplicada entre os dois contatos externos. A diferença de potencial resultante é medida pelos pelos dois contatos internos. 0 equipamento foi calibrado segundo as recomendações do fornecedor antes da realização de cada medida.

Quatro corpos de prova foram utilizados para cada dosagem estabelecida neste artigo, totalizando 44 corpos de prova. As medidas de RES foram obtidas ao posicionar o equipamento nas faces desses corpos de prova.

\subsection{MIGRAÇÃO MULTIREGIME DE CLORETOS}

O método de ensaio multiregime de migração de cloretos proposto pela norma da UNE 83987 (2012) foi seguido neste artigo. Esse método permite obter simultaneamente os valores de $D_{e f}$ e $D_{a p}$ através do uso de uma célula de migração. Essa célula é composta por dois compartimentos: o compartimento do lado catódico é preenchido com uma solução $1 \mathrm{M}$ de $\mathrm{NaCl}$, e o compartimento do lado anódico com água deionizada. Uma diferença de potencial de 12 V DC é aplicada através de barras de aço usadas como eletrodos.

A configuração das células utilizadas neste artigo é semelhante à utilizada por Medeiros et al. (2014). Tubos de PVC foram usados para compor as células de migração de cloretos, conforme Figura 1.

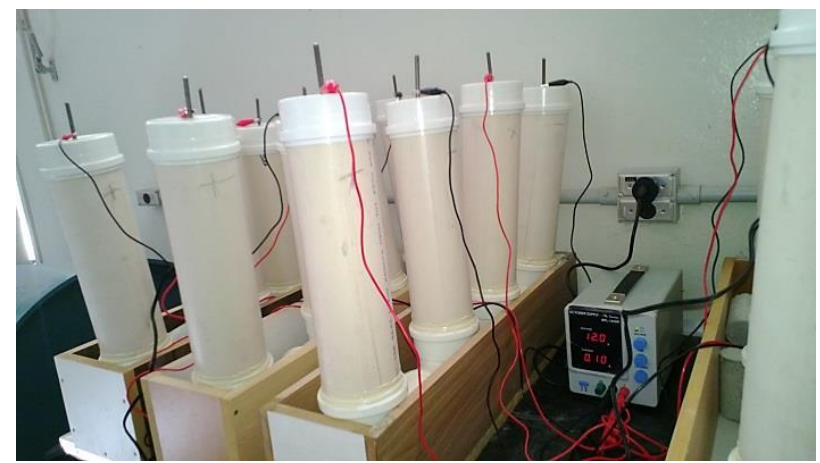

FIGURA 1: Células de migração para ensaio multiregime de migração de cloretos.

FONTE: AUTORES (2014).

As amostras de concreto foram serradas em espessuras de aproximadamente $25 \mathrm{~mm}$. 0 disco de concreto a partir do terço central dos corpos de prova foi utilizado no ensaio, tendo em vista evitar heterogeneidades. Três corpos de prova de cada uma das 11 dosagens de concreto expostas na Tabela 1 foram usados no ensaio de 
migração, totalizando 33 células.

A realização do ensaio conforme a norma

UNE 83987 (2012) permite acompanhar a evolução da concentração de cloretos no compartimento do lado anódico com o tempo, devido à migração de cloretos através do disco de concreto. Segundo essa norma, o coeficiente de difusão efetivo $\left(D_{e f}\right)$ pode então ser calculado pela Equação 1, baseada na equação modificada de Nernst-Planck (ANDRADE, 1993).

$$
\mathrm{D}_{e f}=\frac{R T l}{z A F\left(\gamma C_{C l}\right) \Delta \phi_{e f}} \cdot \frac{d C}{d t}
$$

Onde:

$\mathbf{D}_{\text {ef }}=$ coeficiente de difusão dos cloretos no estado estacionário $\left(\mathrm{cm}^{2} / \mathrm{s}\right)$;

$\mathbf{R}=$ constante dos gases $(=1,9872 \mathrm{cal} /(\mathrm{mol} \cdot \mathrm{K}))$;

$\mathbf{T}=$ temperatura $(\mathrm{K})$;

$\mathrm{I}=$ espessura do disco de concreto $(\mathrm{cm})$;

$\mathbf{z}=$ valência do íon cloreto $(=1)$;

$\mathbf{F}=$ constante de Faraday (=23063 cal/(vol'eq));

$\mathbf{C}_{\mathrm{Cl}}=$ concentração de cloretos na célula catódica $\left(\mathrm{mol} / \mathrm{cm}^{3}\right)$;

$\boldsymbol{\gamma}=$ coeficiente de atividade da solução da célula catódica ( $=0,657$, para cloretos);

$\Delta \boldsymbol{\phi}_{\text {ef }}=$ tensão efetiva que atravessa as amostras durante 0 estado estacionário, monitorada durante o ensaio;

$\mathbf{A}=$ área da seção do disco de concreto disponível para migração dos cloretos $\left(\mathrm{cm}^{2}\right)$; e

$\mathbf{d C} / \mathbf{d t}=$ inclinação da parte linear (estado estacionário) do gráfico (mol/s): "concentração de cloretos x tempo".

Ainda segundo a norma UNE 83987 (2012), o coeficiente de difusão de cloretos aparente $\left(D_{a p}\right)$ é também determinado pelo ensaio multiregime de migração de cloretos, porém considerando o time-lag $\left(t_{t}\right)$, definido como o tempo necessário para os cloretos atravessarem a amostra de concreto, saturando-a (CASTELLOTE, ANDRADE e ALONSO, 2001). Segundo UNE 83987 (2012), $t_{t l}$ é obtido pela interseção da reta característica do fluxo de cloretos no estado estacionário com o eixo " $x$ " (horizontal). Assim, $D_{a p}\left(\mathrm{~cm}^{2} / \mathrm{s}\right)$ é calculado a partir da Equação 2. Na Equação 2, $\phi$ é determinado pela Equação 3 (KEISTER e KASTING, 1986; UNE 83987, 2012).

$$
\begin{aligned}
& \mathrm{D}_{a p}=\frac{l^{2}}{2 \cdot t_{t l} \cdot \varphi} \\
& \varphi=\frac{z \cdot F}{R \cdot T} \cdot \Delta \phi_{a p}
\end{aligned}
$$

Onde:

$\mathbf{D}_{\mathrm{ap}}=$ coeficiente de difusão dos cloretos no estado não estacionário $\left(\mathrm{cm}^{2} / \mathrm{s}\right)$;

$\mathrm{I}=$ espessura do disco de concreto $(\mathrm{cm})$;

$\mathbf{t}_{\mathrm{tl}}=$ time lag $(\mathrm{s})$;

$\boldsymbol{\varphi}=$ coeficiente calculado conforme Equação 3;

$\mathbf{z}=$ valência do íon cloreto $(=1)$;

$\mathbf{F}=$ constante de Faraday (=23063 cal/(voleq));

$\mathbf{R}=$ constante dos gases $(=1,9872 \mathrm{cal} /(\mathrm{mol} \cdot \mathrm{K}))$;

$\mathbf{T}=$ temperatura $(\mathrm{K})$;

$\Delta \boldsymbol{\phi}_{\mathrm{ap}}=$ campo elétrico normalizado $(\mathrm{V} / \mathrm{cm})$, determinado pela Equação 4.

$$
\Delta \phi_{a p}=\frac{\Delta E_{a p}}{l}
$$

Onde:

$\Delta \phi_{\mathrm{ap}}=$ campo elétrico normalizado $(\mathrm{V} / \mathrm{cm})$; $\mathrm{I}=$ espessura do disco de concreto $(\mathrm{cm})$;

$\Delta \mathbf{E}_{\mathrm{ap}}=$ média da voltagem efetiva (V) que atravessa o corpo de prova durante o estado não estacionário do ensaio.

Finalmente, para que $D_{e f}$ e $D_{a p}$ obtidos no método multiregime de migração de cloretos sejam expressos na mesma unidade, é necessário que o valor de $D_{a p}$ calculado pela Equação 2 seja multiplicado pela porosidade da amostra $(\varepsilon$, em \% volume). Segundo UNE 83987 (2012) e Andrade, D’Andréa e Rebolledo (2014), essa conversão é necessária, pois $D_{e f}$ é obtido considerando o fluxo de cloretos na solução dos poros do concreto, e $D_{a p}$ em relação ao volume do sólido como um todo. Neste artigo, a porosidade foi determinada pelo método de intrusão de mercúrio, através de amostras cúbicas de concreto com arestas aproximadas de $1 \mathrm{~cm}$. Essas amostras foram obtidas a partir dos corpos de prova cilíndricos moldados para cada dosagem investigada.

Além da determinação dos coeficientes de difusão, a corrente elétrica que passa pelos corpos de prova de concreto durante o ensaio multiregime de migração de cloretos foi periodicamente monitorada (a cada $48 \mathrm{~h}$ ), por meio 
do uso de um multímetro, conforme mostra a Figura 2.

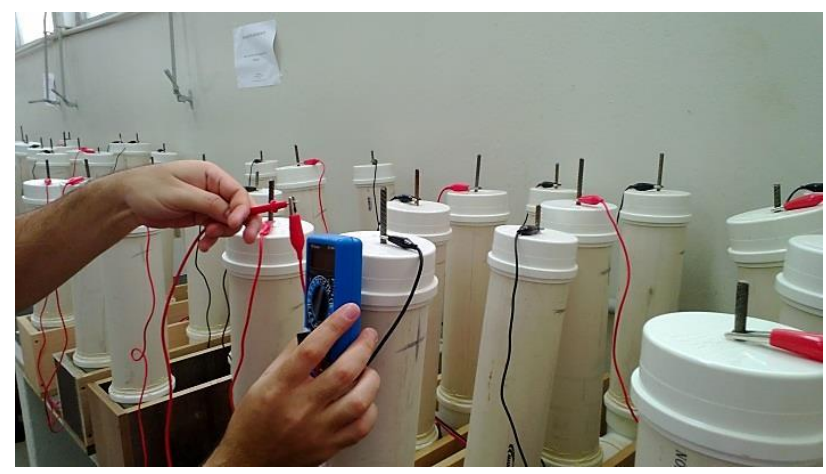

FIGURA 2: Medição da corrente elétrica durante ensaio multiregime de migração de cloretos.

FONTE: AUTORES (2014).

\section{RESULTADOS E DISCUSSÕES}

\subsection{CARACTERIZAÇÃO INICIAL DAS AMOSTRAS DE CONCRETO}

A Tabela 2 apresenta os resultados do ensaio de consistência do concreto fresco, resistência à compressão aos 28 dias, e resistividade elétrica superficial, também aos 28 dias.

Segundo a Tabela 2, verifica-se que o abatimento do concreto fresco variou entre as amostras. Essa variação foi influenciada não apenas pela relação a/c, mas também pelo tipo de cimento utilizado. No geral, foi verificado que para relação água/cimento $(\mathrm{a} / \mathrm{c})$ igual a 0,4 , todas as amostras apresentaram abatimento igual à zero. No entanto, para relação a/c igual a 0,5, observouse que os cimentos CPII-F e CPIV proporcionaram um abatimento na faixa de 145 a $165 \mathrm{~mm}$, diferentemente dos cimentos CPIIIRS e CPV, onde esses valores foram na faixa de 90 a $95 \mathrm{~mm}$. Já para a relação a/c igual a 0,6 , o concreto com o cimento CPIV foi o que apresentou menor abatimento, igual a $200 \mathrm{~mm}$. As outras amostras obtiveram abatimento na faixa de 230 a $250 \mathrm{~mm}$.

Um fator que deve ser levado em consideração devido às diferentes quantidades de água e tipo de cimento entre as amostras é a interferência na resistência mecânica do concreto. Os resultados da Tabela 2 comprovam que a resistência à compressão diminui com o aumento da relação a/c do concreto. Esse comportamento também foi identificado em outros estudos (MEHTA e MONTEIRO, 2005; MEDEIROS-JUNIOR et al., 2014). Conforme a Tabela 2, os tipos de cimentos tem influência na resistência do concreto. Foi observado que os concretos compostos por cimentos com maiores teores de adições (isto é, CPIV e CPIIIRS) apresentaram menores resistência à compressão, concordando com outros estudos (KHATIB e HIBBERT, 2005; ONER e AKYUZ, 2007; LÜBECK et al., 2012). Portanto, segundo os resultados, de maneira geral, para uma mesma relação $a / c$, a resistência à compressão cresce à medida que são utilizados os seguintes tipos de cimento: CPIV, CPIIIRS, CPII-F e CPV.

A Tabela 2 também indica que a resistividade elétrica superficial (RES) decresce à medida que a relação a/c aumenta, quando analisado um mesmo tipo de cimento. Esse comportamento é explicado pelo fato da água funcionar como um condutor no interior do concreto. Dessa forma, quanto maior a quantidade de água no concreto, combinado com a maior permeabilidade do meio, ambos provenientes da maior relação a/c, tem-se uma maior condutividade no interior do material, resultando em menores valores de RES. Esses resultados estão em acordo com Olsson et al. (2013).

Foi observado que a RES para os concretos com cimento CPIIIRS foram superiores a RES das outras amostras. Tal comportamento pode ser atribuído à quantidade de adições de escórias de alto forno na composição do cimento CPIIIRS ( 60\%). Essas adições contribuem para refinar os poros da microestrutura do concreto, e reduzir a permeabilidade das amostras. Consequentemente, a condutividade das amostras é menor, e a RES se eleva.

Page, Lambert e Vassie (1991) comentam que cimentos contendo escória ou pozolanas restringem a mobilidade, seja de corrente elétrica ou de íons, dentro do concreto. Isto ocorre porque estes cimentos reduzem substancialmente a permeabilidade do material, o que reflete na elevação da RES.

Corroborando com as afirmações 
anteriores, segundo a Tabela 2, as amostras com cimento CPIV foram as que obtiveram segundo melhor desempenho em termos de RES, comportamento explicado pela quantidade de adições pozolônicas na composição do cimento ( 50\%). Já os cimentos CPII-F e CPV apresentaram valores inferiores de RES porque têm poucas adições nas suas composições. Esses resultados estão em concordância com a literatura. Gesoğlu e Özbay (2007) verificaram valores mais elevados de RES em amostras contendo adições de escórias quando comparadas com concreto sem nenhuma adição. Já Lübeck et al. (2012) observaram um aumento de 2,52 a 4,76 vezes na RES aos 7 dias de idade de amostras de concreto após substituição de $50 \%$ do cimento Portland comum por escória.

\subsection{COEFICIENTES DE DIFUSÃO}

A Figura 3 apresenta os coeficientes de difusão efetivo $\left(D_{e f}\right)$ e aparente $\left(D_{a p}\right)$, bem como a relação encontrada entre eles.

De acordo com os resultados, $D_{\text {ef }}$ varia entre $5 \times 10^{-9}$ a $6 \times 10^{-8} \mathrm{~cm}^{2} / \mathrm{s}$ e $D_{a p}$ varia entre $3,5 \times 10^{-9}$ a $1,7 \times 10^{-8} \mathrm{~cm}^{2} / \mathrm{s}$. Segundo a Figura 3,0 coeficiente de difusão efetivo é aproximadamente 4,2 vezes o coeficiente aparente. Uma boa correlação linear foi encontrada entre os dados, com coeficiente de determinação igual a 0,97. Esses resultados são interessantes, contudo, a relação foi obtida através de um ajuste de correlação, baseado na obtenção de um maior valor de $\mathrm{R}^{2}$ possível, e considera todos os cimentos investigados juntos. Deve-se levar em consideração que essa relação é válida para as amostras estudadas neste artigo, e pode variar para outras composições de materiais e condições de ensaios. Medeiros-Junior (2014) verificou que ao analisar separadamente cada tipo de cimento, a relação entre os coeficientes de difusão varia em um intervalo de 1,3 a 4,0.

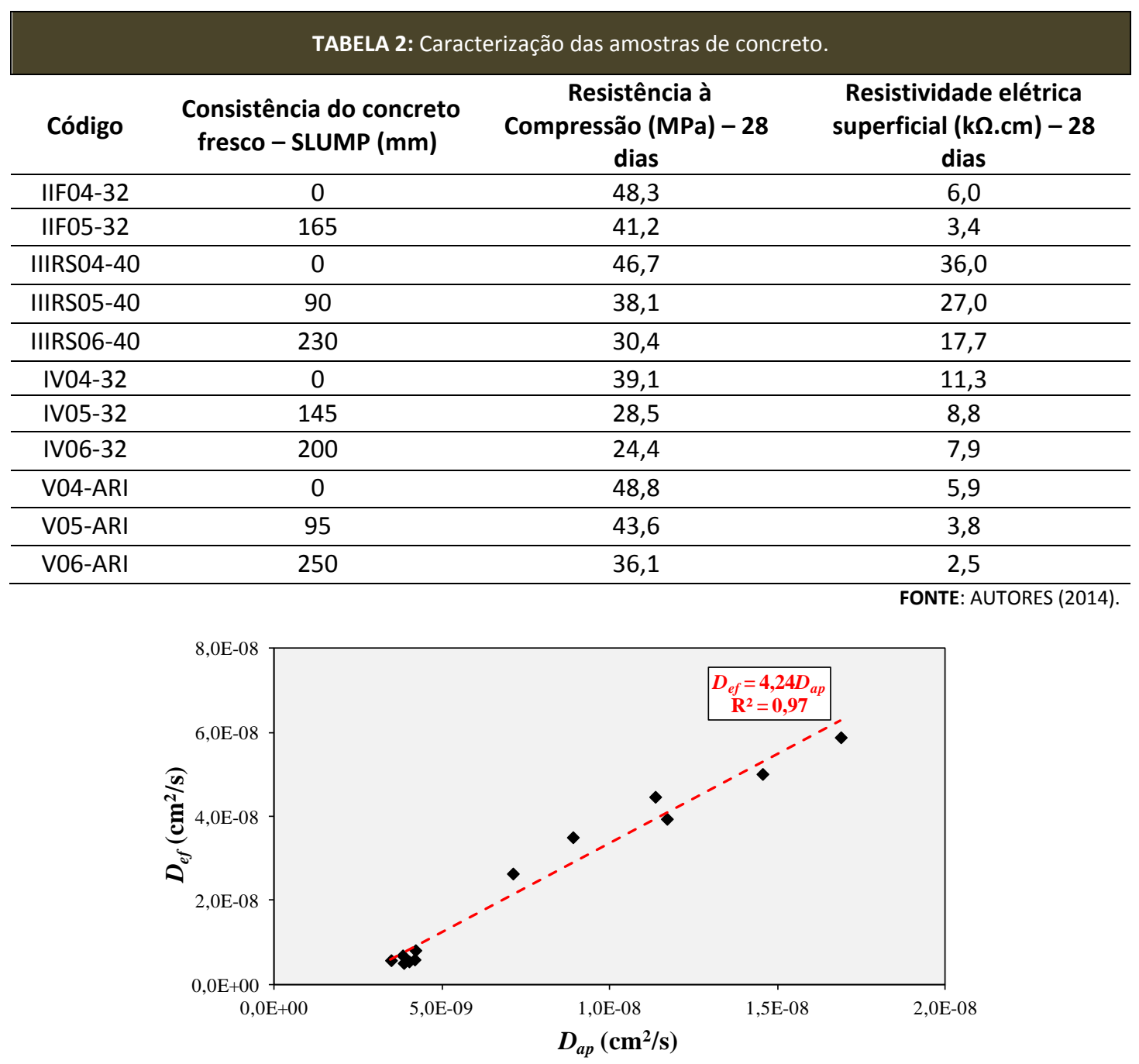

FIGURA 3: Relação entre $D_{e f}$ e $D_{a p}$ para todos os tipos de cimento. FONTE: AUTORES (2014). 


\subsection{CORRENTE ELÉTRICA}

O comportamento da corrente elétrica identificado para todas as amostras durante o ensaio de migração de cloretos pode ser resumido da seguinte maneira: há um momento de crescimento da corrente elétrica até a mesma atingir um valor máximo durante o ensaio (pico); posteriormente, a corrente começa a diminuir até se estabilizar. Medeiros e Lima (2014) demonstraram esse comportamento.

É interessante registrar que esses picos de corrente tem intensidades diferentes de acordo com o tipo de cimento investigado. A Tabela 3 apresenta os valores do pico de corrente $(\mathrm{mA})$ para as diferentes amostras testadas.

Além dos tipos de cimentos, observa-se que a relação a/c também influência no valor do pico de corrente. Esse comportamento indica que a corrente elétrica obteve maior facilidade em percorrer através dos poros dos concretos com maior relação a/c, sugerindo maior permeabilidade dessas amostras. Santos (2006) também verificaram essa tendência. De acordo com Mehta e Monteiro (2005), os concretos com maior relação a/c possuem maior quantidade de solução com cloretos nos poros, devido à maior permeabilidade resultante do aumento da relação a/c.

Além do comentado, Medeiros-Junior e Lima (2014) discutem que nos concretos com maior relação $a / c$, os íons se encontram mais dissolvidos, favorecendo a mobilidade iônica da solução. Em geral, em concentrações menos concentradas, os íons apresentam maior facilidade de se locomover no interior do concreto, conforme John e Cincotto (1995). Esses dois fatores contribuem para o aumento do pico da corrente elétrica em concretos com maiores relação a/c.

Assim como observado no ensaio de RES aos 28 dias, o pico de corrente é menor para as amostras de concretos com cimentos com maiores teores de adições (isto é, CPIIIRS e CPIV), devido também à microestrutura mais densa gerada por esses tipos de concreto, o que diminui a condutividade no interior do concreto. Ao considerar a relação a/c igual a 0,5, por exemplo, a Tabela 3 mostra que o pico de corrente foi igual a 20,$7 ; 27,7 ; 47,8$; e 47,9 ; para as amostras com os cimentos CPIIIRS, CPIV, CPV e CPII-F, respectivamente.

TABELA 3: Pico de corrente para as amostras de concreto.

\begin{tabular}{lc} 
Código & $\begin{array}{c}\text { Pico de corrente médio (mA) } \pm \text { desvio } \\
\text { padrão }\end{array}$ \\
\hline IIF04-32 & $33,5 \pm 4,5$ \\
\hline IIF05-32 & $47,9 \pm 1,6$ \\
\hline IIIRS04-40 & $17,5 \pm 0,5$ \\
\hline IIIRS05-40 & $20,7 \pm 11,2$ \\
\hline IIIRS06-40 & $21,3 \pm 1,3$ \\
\hline IV04-32 & $27,7 \pm 3,9$ \\
\hline IV05-32 & $27,7 \pm 3,6$ \\
\hline IV06-32 & $31,2 \pm 4,7$ \\
\hline V04-ARI & $34,3 \pm 3,9$ \\
\hline V05-ARI & $47,8 \pm 8,2$ \\
\hline V06-ARI & $53,9 \pm 6,3$ \\
\hline
\end{tabular}




\subsection{CORRELAÇÃO ENTRE OS COEFICIENTES DE DIFUSÃO E A CORRENTE ELÉTRICA}

A seguir são reveladas correlações entre 0 pico de corrente $\left(I_{\text {pico }}\right)$ medido durante o ensaio de migração de cloretos e o coeficiente de difusão efetivo (Figura 4), e a mesma relação para o coeficiente de difusão aparente (Figura 5).

O melhor ajuste encontrado para os dados das Figuras 4 e 5 foi do tipo linear. Conforme essas figuras, o pico de corrente é melhor relacionado com $D_{e f}$ do que com $D_{a p}$ baseado no melhor valor de $\mathrm{R}^{2}$ identificado.

Baseado nos resultados deste estudo, as
Equações 5 e 6 permitem correlacionar os valores de pico de corrente e os coeficientes de difusão de cloretos. Esses resultados são de extrema relevância, pois contribuem para facilitar a obtenção de variáveis importantes para a modelagem dos cloretos no concreto, como os coeficientes de difusão. Porém, ressalta-se que para o uso das equações mencionadas com prudência, recomenda-se adotar as condições de ensaio e amostras estabelecidas neste artigo, e verificar se a precisão $\left(R^{2}\right)$ encontrada neste estudo atende a aplicação requerida para os dados.

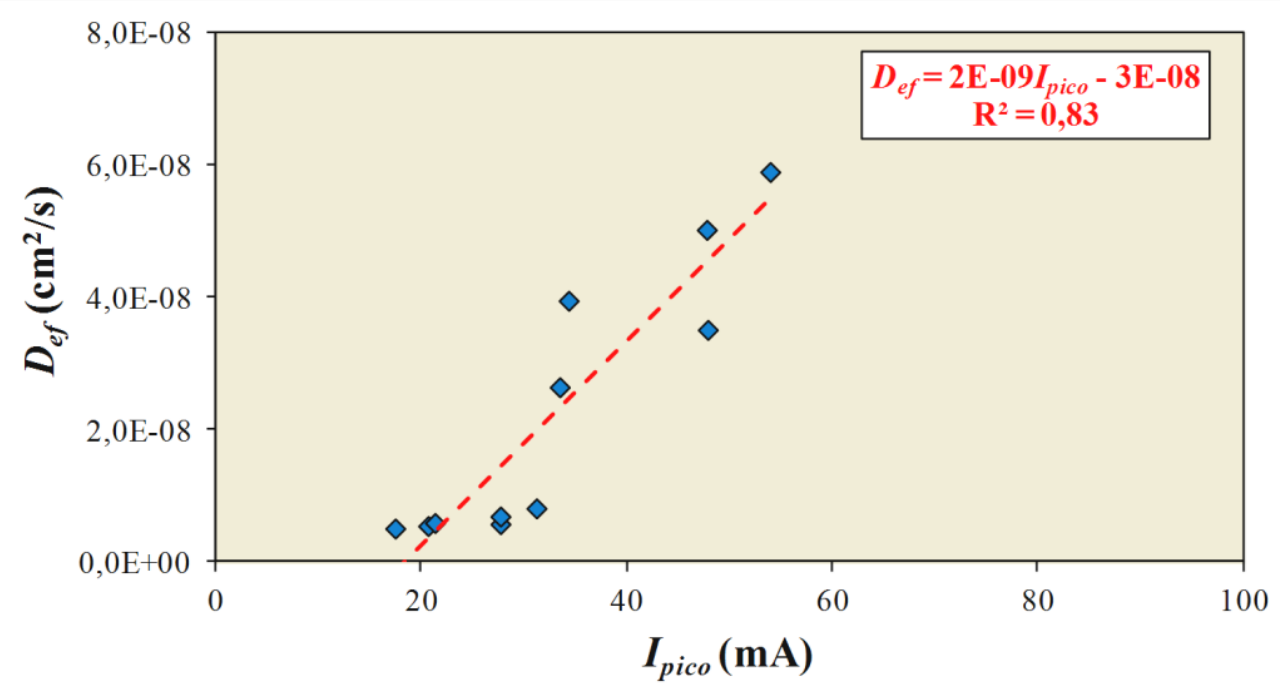

FIGURA 4: Relação entre os coeficientes de difusão de cloretos e o pico de corrente durante ensaio de migração $-D_{e f}$.

FONTE: AUTORES (2014).

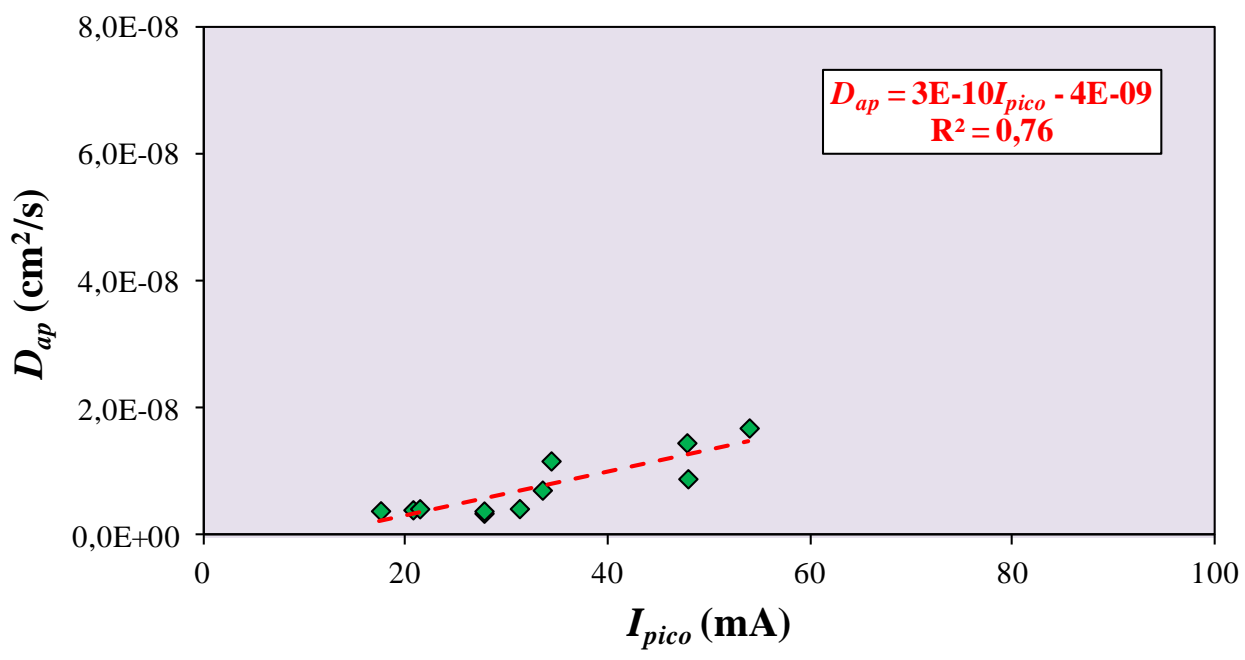

FIGURA 5: Relação entre os coeficientes de difusão de cloretos e o pico de corrente durante ensaio de migração - $D_{a p}$. FONTE: AUTORES (2014). 


$$
\begin{array}{ll}
\mathrm{D}_{e f}=\left(2 \times 10^{-9} \cdot I_{\text {pico }}\right)-3 \times 10^{-8} & \text { Eq. [5] } \\
\mathrm{D}_{a p}=\left(3 \times 10^{-10} \cdot I_{\text {pico }}\right)-4 \times 10^{-9} & \text { Eq. [6] }
\end{array}
$$

Onde:

$\mathbf{D}_{\text {ef }}=$ coeficiente de difusão dos cloretos efetivo estado estacionário $\left(\mathrm{cm}^{2} / \mathrm{s}\right)$;

$\mathbf{D}_{\mathrm{ap}}=$ coeficiente de difusão dos cloretos aparente - estado não estacionário $\left(\mathrm{cm}^{2} / \mathrm{s}\right)$;

$\mathbf{I}_{\text {pico }}=$ pico de corrente durante ensaio multiregime de migração de cloretos (mA).

\section{CONSIDERAÇÕES FINAIS}

Este estudo identificou correlações entre o pico de corrente durante o ensaio multiregime de migração de cloretos, e os coeficientes de difusão em estado estacionário e não estacionário. Quatro tipos de cimentos foram investigados, com diferentes relações a/c. Para as amostras analisadas, os resultados mostraram que os coeficientes de difusão efetivos são cerca de 4,2 vezes o coeficiente de difusão aparente. Além disso, tanto os coeficientes de difusão, quanto o pico de corrente durante o ensaio de migração, são menores para as amostras de concreto com cimentos com altos teores de adições, tais como escória de alto forno e pozolanas, o que indica menor permeabilidade para essas amostras. Isso também foi confirmado nos resultados do ensaio de resistividade elétrica superficial. Finalmente, as correlações encontradas neste artigo contribuem para a obtenção de parâmetros importantes para a modelagem de cloretos no concreto.

\section{AGRADECIMENTOS}

Os autores agradecem a Fundação de Amparo à Pesquisa do Estado de São Paulo (FAPESP) por fornecer apoio financeiro na elaboração deste estudo e ao Instituto Tecnológico de Aeronáutica (ITA) por providenciar a infraestrutura necessária.

\section{REFERÊNCIAS BIBLIOGRÁFICAS}

ANDRADE, C. Calculation of chloride diffusion coefficients in concrete from ionic migration measurements. Cement and Concrete Research, Vol. 23, 1993, 724-742 p.

ANDRADE, C. Manual para diagnóstico de obras deterioradas por corrosão de armaduras. São Paulo: Pini, 1992, $104 \mathrm{p}$.

ANDRADE, C.; D'ANDRÉA, R. Electrical resistivity as microstructural parameter for the modeling of service life of reinforced concrete structures. In.: 2nd International Symposium on Service Life Design for Infrastructure. Delft, The Netherlands, 2010, 379-388 p.

ANDRADE, C.; D’ANDRÉA, R.; REBOLLEDO, N. Chloride ion penetration in concrete: the reaction factor in the electrical resistivity model. Cement and Concrete Composites, Vol. 47, 2014, 41-46 p.

ANDRADE, C.; SANJUÁN, M. A.; RECUERO, A.; RÍO, O. Calculation of chloride diffusivity in concrete from migration experiments, in non steady-state conditions. Cement and Concrete Research, Vol. 24, n. 7, 1994, 1214-1228 p.

ANDRADE, C.; WHITING, D. A comparison of chloride ion diffusion coefficients derived from concentration gradients and non-steady state accelerated ionic migration. Materials and Structures, Vol. 29, 1996, 476$484 \mathrm{p}$.

ASSOCIAÇÃO BRASILEIRA DE NORMAS TÉCNICAS, NBR 5733: Cimento Portland de alta resistência inicial. Rio de Janeiro, 1991.

NBR 5735: Cimento Portland de alto-forno. Rio de Janeiro, 1991.

NBR 5736: Cimento Portland pozolânico. Rio de Janeiro, 1991.

NBR 5737: Cimentos Portland resistentes a sulfatos. Rio de Janeiro, 1992.

NBR 5739: Concreto - Ensaios de compressão de corpos-de-prova cilíndricos. Rio de Janeiro, 2007.

NBR 11578: Cimento Portland composto Especificação. Rio de Janeiro, 1991.

NBR NM 30: Agregado miúdo - Determinação da absorção de água. Rio de Janeiro, 2001.

NBR NM 46: Agregados - Determinação do material fino que passa através da peneira $75 \mu \mathrm{m}$, por lavagem. Rio de Janeiro, 2003. 
NBR NM 52: Agregado miúdo - Determinação da massa específica e massa específica aparente. Rio de Janeiro, 2009.

NBR NM 53: Agregado graúdo - Determinação da massa específica, massa específica aparente e absorção de água. Rio de Janeiro, 2009.

NBR NM 248: Agregados - Determinação da composição granulométrica. Rio de Janeiro, 2003.

ATKINSON, A.; NICKERSON, A. K. The diffusion of ions through water-saturated cement. Journal of Materials Science, Vol. 19, 1984, 3068-3078 p.

CASTELLOTE, M.; ANDRADE, C.; ALONSO, C. Measurement of the steady and non-steady-state chloride diffusion coefficients in a migration test by means of monitoring the conductivity in the anolyte chamber - comparison with natural diffusion tests. Cement and Concrete Research, Vol. 31, 2001, 1411 $1420 \mathrm{p}$

GESOĞLU, M.; ÖZBAY, E. Effects of mineral admixtures on fresh and hardened properties of self-compacting concretes: binary, ternary and quaternary systems. Materials and Structures, Vol. 40, n. 9, 2007, 923-937 p.

GOWERS, K. R.; MILLARD, S. G. Measurement of concrete resistivity for assessment of corrosion severity of steel using wenner technique. $\mathrm{ACl}$ Materials Journal, Vol. 96-M66, 1999, 536-541 p.

HELENE, P. R. L. Contribuição ao estudo da corrosão em armaduras de concreto armado. Tese (Livre Docência) - Escola Politécnica da Universidade de São Paulo, Departamento de Engenharia Civil, São Paulo, 1993, $271 \mathrm{p}$

JOHN, V. M.; CINCOTTO, M. A. Escórias de alto forno como aglomerante. BT/PCC/152 Boletim Técnico da Escola Politécnica da USP. Departamento de Engenharia de Construção Civil, São Paulo, 1995, 1-25 p.

KEISTER, J. C.; KASTING, G. B. Ionic mass transport through a homogeneous membrane in the presence of a uniform electrical field. Journal of Membrane Science, Vol. 29, 1986, 155-167 p.

KHATIB, J.; HIBBERT, J. J. Selected engineering properties of concrete incorporating slag and metakaolin. Construction and Building Materials, Vol. 19, 2005, 460-472 p.

LÜBECK, A.; GASTALDINI, A. L. G.; BARIN, D. S.; SIQUEIRA, H. C. Compressive strength and electrical properties of concrete with white Portland cement and blast-furnace slag. Cement and Concrete Composites, Vol. 34, 2012, 392-399 p.
MEDEIROS, M. H. F.; REAL, L. V.; RICHTER, K.; SOUZA, W. B.; KLEIN, N. S. Ensaios de migração de cloretos em estado estacionário para avaliação de sistemas de proteção de superfície. Revista Eletrônica de Engenharia Civil, Vol. 8, 2014, 54-63 p.

MEDEIROS-JUNIOR, R. A. Estudo da influência das mudanças climáticas na penetração de ions cloretos em estruturas de concreto localizadas em ambiente marinho. Dissertação (mestrado) - Programa de PósGraduação em Engenharia de Infraestrutura Aeronáutica. Instituto Tecnológico de Aeronáutica ITA. São José dos Campos, São Paulo, 2011, 191 p.

MEDEIROS-JUNIOR, R. A. Estudo da resistividade do concreto para proposta de modelagem de vida útil Corrosão das armaduras devido à penetração de cloretos. Tese (doutorado) - Programa de PósGraduação em Engenharia de Infraestrutura Aeroportuária - Instituto Tecnológico de Aeronáutica (ITA), São José dos Campos-SP, 2014, 243 p.

MEDEIROS-JUNIOR, R. A.; LIMA, M. G. Comportamento da corrente elétrica durante ensaio de migração de cloretos em diferentes amostras de concreto. In: X Congreso Internacional sobre Patología y Recuperación de Estructuras-CINPAR. Santiago, Chile, 2014, 1-9 p.

MEDEIROS-JUNIOR, R. A.; LIMA, M. G.; MEDEIROS, M. H. F.; REAL, L. V. Investigação da resistência à compressão e da resistividade elétrica de concretos com diferentes tipos de cimento. Revista ALCONPAT, Vol. 4, 2014, 116-132 p.

MEHTA, P. K.; MONTEIRO, P. J. M. Concrete: microstructure, properties and materials. 3a $\mathrm{Ed}$. McGraw-Hill Professional, 2005. 660 p.

MEIRA, G. R. Agressividade por cloretos em zona de atmosfera marinha frente ao problema da corrosão em estruturas de concreto armado. Tese (doutorado) Programa de Pós-Graduação em Engenharia Civil PPGEC. Universidade Federal de Santa Catarina - UFSC, Florianópolis-SC, 2004, 369 p.

NORMA ESPAÑOLA, UNE 83987: Determinación de los coeficientes de difusión de los iones cloruro en el hormigón endurecido. Método multiregimen. Espanha, 2012.

OLSSON, N.; BAROGHEL-BOUNY, V.; NILSSON, L.; THIERY, M. Non-saturated ion diffusion in concrete - $A$ new approach to evaluate conductivity measurements. Cement and Concrete Composites, Vol. 40, 2013, 40-47 p.

ONER, A. E.; AKYUZ, S. An experimental study on optimum usage of GGBS for the compressive strength of concrete. Cement and Concrete Composites, Vol. 29, 2007, 505-514 p. 
PAGE, C. L.; LAMBERT, P.; VASSIE, P. R. W. Investigation of reinforcement corrosion. Part 1: The pore electrolyte phase in chloride contaminated concrete. Materials and Structures, Vol. 24, 1991, 243-252 p.

RAMLI, M.; TABASSI, A. A. Influences of polymer modification and exposure conditions on chloride permeability of cement mortars and composites. Journal of Materials in Civil Engineering, Vol. 24, n. 2, 2012, 216-222 p.

REDDY, D. V.; EDOUARD, J.; SOBHAN, K. Durability of fly ash-based geopolymer structural concrete in the marine environment. Journal of Materials in Civil Engineering, Vol. 25, n. 6, 2013, 781-787 p.

SANTOS, L. Avaliação da resistividade elétrica do concreto como parâmetro para a previsão da iniciação da corrosão induzida por cloretos em estruturas de concreto. Dissertação (mestrado) - Universidade de Brasília - UNB. Brasília, Distrito Federal, 2006, 161 p. 